\title{
EL CONFLICTO MAPUCHE EN LA PRENSA CHILENA. ANOTACIONES TEÓRICO- METODOLOGICAS PARA EL ANÁLISIS DE LOS ENCUADRES MEDIÁTICOS' ${ }^{1}$
}

\author{
Dra. Nadia Koziner \\ CONICET-UNQ, Ciudad Autónoma de Buenos Aires, Argentina \\ nadiakoziner@gmail.com \\ ORCID iD: https://orcid.org/0000-0003-2834-4799 \\ Dra. Natalia Aruguete \\ CONICET-UNQ, Ciudad Autónoma de Buenos Aires, Argentina \\ nataliaaruguete@gmail.com \\ ORCID iD: https://orcid.org/0000-0002-1571-9224
}

Recibido el 28 de abril de 2020

Aceptado el 20 de mayo de 2020

\section{Resumen}

Este trabajo aporta un planteo teórico-metodológico para detectar y conceptualizar los encuadres del conflicto entre las comunidades Mapuche y el Estado chileno, que quedan plasmadas en las noticias de la prensa gráfica nacional y regional. Nuestro propósito es sentar las bases epistemológicas y conceptuales sobre las cuales construimos una matriz para el análisis de los textos. La revisión de la literatura es una pieza clave para encontrar una definición operacional que pueda desagregarse en dimensiones $y$, dentro de estas, en variables que pongan de manifiesto los marcos interpretativos del conflicto mapuche en los medios chilenos.

Palabras clave: encuadres mediáticos, conflicto mapuche, prensa chilena, análisis de contenido.

\footnotetext{
${ }^{1}$ Este artículo fue realizado en el marco del proyecto PLU 170002, titulado "Diseño de un indicador de pluralismo aplicado a la tematización de la agenda de la prensa escrita y a la validación de las fuentes. Caso de estudio: La cobertura del tema mapuche en la prensa nacional y en la prensa regional" y fue dirigido por Eileen Hudson Frías, de la Universidad del Desarrollo (UDD). Participaron como coinvestigadores Carlos del Valle Rojas, de la Universidad de la Frontera (UFRO); Francisca Dussaillant (UDD); Rodrigo Browne, de la Universidad Austral de Chile (UACh), y como investigadoras extranjeras, Natalia Aruguete, del CONICET y la Universidad aNacional de Quilmes, Argentina, y Nadia Koziner, del CONICET y la Universidad de Buenos Aires y de la Universidad Nacional de Quilmes, Argentina.
} 
THE MAPUCHE CONFLICT IN THE CHILEAN PRESS. THEORETICALMETHODOLOGICAL ANNOTATIONS FOR MEDIA FRAMES ANALYSIS

\begin{abstract}
This work provides a theoretical-methodological approach to detect and conceptualize the framings of the conflict between the Mapuche communities and the Chilean State which are expressed in the news of the national and regional press. Our purpose is to lay the epistemological and conceptual bases on which we build a matrix to analyze texts. The literature review is a key piece to find an operational definition that can be disaggregated into dimensions and, within these, into variables that highlight the interpretative frameworks of the Mapuche conflict in the Chilean media.
\end{abstract}

Keywords: news frames, mapuche conflict, Chilean press, content analysis. 


\title{
1. Introducción
}

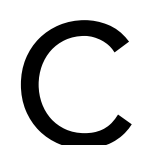

uando realizamos estudios empíricos, más aún cuando procuramos reflexionar en torno a la dimensión teórico-epistemológica de un objeto de estudio, una de las principales dificultades surge en la instancia de delimitación del problema de investigación. Es decir que más allá de la relevancia política y académica que amerite la investigación de un fenómeno, corremos el riesgo de encontrarnos con grandes temas de interés que no son directamente abordables dado su grado de abstracción, complejidad y amplitud. En efecto, en el marco de un mismo tema, es posible formular una infinidad de preguntas que derivan en problemas de investigación distintos.

\begin{abstract}
Metafóricamente se podría pensar esta cuestión como un recorrido espiralado descendente en el que los anillos de la espiral se hacen cada vez más pequeños, representando de este modo la mayor focalización gradual que van adquiriendo los asuntos de interés, hasta llegar a un núcleo que constituye el problema de investigación (Piovani, 2018: 90).
\end{abstract}

En nuestro caso, la intención de delimitar un objeto de estudio que se derive del tema mapuche en Chile nos lleva a estudiar la puja por instalar una definición de la situación alrededor del conflicto entre el Estado nacional chileno y las comunidades Mapuche de ese país, así como sus causas y los modos de resolverlo. El procedimiento que encontramos para ir de lo abstracto y general del tema hacia lo específico y concreto del problema, consiste en focalizar la atención en los territorios físicos y simbólicos en los cuales esas disputas tienen lugar. En este punto, cabe atender al rol que la prensa gráfica, y especialmente los diarios nacionales y regionales de las zonas en conflicto, han desempeñado en la coconstrucción de un sentido común en relación con esta cuestión (Valle, 2005). De allí que nuestro objeto se centre en las formas en las que los encuadres ${ }^{2}$ del conflicto mapuche se manifiestan en los textos noticiosos.

La inquietud por dar cuenta de los modos en que los discursos de las noticias les dan sentido a determinados conflictos políticos no es nueva. En trabajos previos, nos hemos enfocado en la operacionalización de las distintas nociones de "encuadre" y "encuadrar", con la intención de alcanzar precisión en el diseño de variables y categorías válidas y confiables que nos permitan conocer los textos mediáticos y volver asequible la "caja negra metodológica" a la que se refieren Matthes y Kohring (2008: 262). Para ello, pasamos de la aplicación deductiva de encuadres genéricos que hemos reconfigurado a partir de su fiabilidad interna (Aruguete y Koziner, 2014; Koziner y Aruguete, 2016), a la operacionalización de la definición propuesta por Entman (1993) en diálogo con casos de relevancia política

2 En este trabajo, usaremos los términos "frame", "encuadre", "marco" y "enfoque" indistintamente. 
(Aruguete y Amadeo, 2012; Koziner, 2017a; Koziner y Aruguete, 2017). Ello implicó un esfuerzo doble. Por un lado, avanzar progresivamente en el afinamiento de las nociones conceptuales y abstractas para arribar a la definición de dimensiones y variables observables en los textos. Por otro, reconocer e incorporar a dicha operacionalización las singularidades de los casos bajo estudio en lugar de realizar una aplicación acrítica de variables definidas para otros estudios, en otras latitudes. Sin embargo, en ese esmero por traducir los conceptos en indicadores observables, hemos prestado menor atención a la reflexión teórico-epistemológica profunda de la base que sustenta conceptualmente esas variables.

De allí que el objetivo de este trabajo consista en un planteo teórico metodológico que nos permita detectar y conceptualizar los encuadres del conflicto entre las comunidades Mapuche y el Estado chileno, que quedan de manifiesto en las noticias de la prensa gráfica nacional y regional. En otras palabras, nos proponemos sentar las bases epistemológicas y teóricas sobre las cuales se construye la matriz de análisis de los textos. Para ello, revisamos la literatura con el fin de encontrar una definición operacional que nos permita desagregar dimensiones y, dentro de estas, variables que pongan de manifiesto los marcos interpretativos del conflicto mapuche en los medios.

Con este objetivo, proponemos seguir el siguiente recorrido. En primer lugar, llevamos a cabo una reflexión teórico-metodológica acerca del diseño de nuestra investigación; en segundo lugar, desarrollamos cada una de las dimensiones que componen la matriz de análisis a partir de la operacionalización del concepto de "encuadre" propuesto por Entman (2004); por último, presentamos las reflexiones finales que se derivan del procedimiento analítico alrededor del diseño de la matriz.

\section{Diseño de la investigación}

El procedimiento teórico-metodológico que nos planteamos para avanzar en el diseño de nuestra investigación incluye la delimitación del corpus, la definición de las unidades de análisis y la operacionalización del concepto de "encuadre" propuesto por Entman, en una serie de dimensiones que surgen del diálogo entre la perspectiva teórica y el caso bajo análisis.

Los hallazgos de trabajos previos funcionan como premisas de investigación que podrán ser ampliadas o refutadas aquí. Por caso, el estudio de Carlos del Valle Rojas (2005) constituye un antecedente relevante para el presente trabajo, por cuanto sistematiza un conjunto de regularidades empíricas que abordan la representación del pueblo Mapuche en el discurso periodístico: sus integrantes suelen ser representados de forma negativa cuando asumen roles activos y valorados en términos positivos toda vez que se les adjudica roles pasivos, como en la recepción de ayuda gubernamental. Además, la presencia de lo mapuche en la prensa se limita al relato de distintos tipos de "conflictos", al tiempo que se recurre a las fuerzas policiales y a políticos como las fuentes autorizadas, legítimas y creíbles. 


\subsection{Corpus y unidades de análisis}

El universo de análisis del presente estudio está conformado por todas las notas de los géneros informativo y argumentativo —unidades de análisis de este trabajopublicadas por cinco diarios (El Mercurio, La Tercera, El Diario de Concepción, El Austral de los Ríos y El Austral de la Araucanía), durante los dos primeros años del primer mandato de Sebastián Piñera como Presidente de la Nación (del 11 de marzo de 2010 al 10 de marzo de 2012) y los dos primeros años del segundo gobierno de Michelle Bachelet (del 11 de marzo de 2014 al 10 de marzo de 2016). En trabajos previos (Koziner y Aruguete, 2020) se tomó la decisión de utilizar el artículo como unidad de análisis, atendiendo a que un mismo párrafo puede entrañar distintos elementos de encuadre, en tanto otros pueden no presentar ninguno. Si bien la unidad de análisis contempla aquello sobre lo cual recae la mirada comprensiva del estudio, las unidades de registro varían en función del elemento de encuadre $y$, más concretamente, de la variable que se esté codificando.

En este punto, es preciso discriminar entre las distintas unidades de análisis. Krippendorff (1990) distingue las unidades de muestreo de las unidades de registro. Las unidades de muestreo son unidades físicas, es decir porciones de la realidad observadas que tienen independencia lógica entre sí en el sentido de que su inclusión o exclusión no tiene consecuencias sobre las otras unidades de muestreo. Dado que son demasiado amplias y complejas para describir directamente la presencia de las variables manifiestas en el texto, se recurre a unidades de registro, segmentos específicos del texto que toman valores al situarlo en una categoría determinada (Holsti, 1969). Estas unidades no son independientes unas de otras, aunque es posible describirlas individualmente según la posición que asumen dentro de las relaciones con otras unidades de registro.

El primer paso para recolectar las notas que serían efectivamente incorporadas al corpus de análisis fue revisar las secciones informativas y argumentativas de los diarios. La selección de una pieza periodística — fuere esta de género informativo o argumentativo- como parte del corpus se llevó a cabo a partir de la detección de una o más nociones clave en el título, en la bajada o en alguno de los tres primeros párrafos de la nota. En aquellos casos en que estos fragmentos del texto no proporcionaran la información suficiente, se revisó la nota completa. Por "nociones clave" se entienden todas aquellas palabras o términos que se refieren explícita o implícitamente a la cuestión mapuche, en cualquiera de sus aspectos.

\subsection{Reflexiones acerca del procedimiento analítico de los encuadres noticiosos}

Para el análisis de los encuadres noticiosos de la cuestión mapuche proponemos dos etapas. En un primer momento, reflexionamos acerca del procedimiento apropiado para la operacionalización de la definición de encuadre. En la segunda instancia, avanzamos en el diseño de una matriz que nos permitiera abordar de 
manera comprensiva los marcos interpretativos que entran en disputa en la definición del conflicto mapuche y conocer cuáles logran quedar instalados como legítimos.

En la primera fase, recurrimos al planteo epistemológico que realizan Matthes y Kohring (2008) para el análisis de los encuadres de la biotecnología en la prensa estadounidense. Ello incluye la recolección de los datos, su procesamiento y la posterior creación de los encuadres del caso.

Partiendo de la clásica definición de Entman (1993), estos autores estudiaron las características propias del fenómeno e identificaron los núcleos temáticos en torno a los cuales se organizaba el debate público. De igual modo, en el presente trabajo, el primer paso para la conceptualización de las dimensiones y variables que componen la matriz de análisis consiste en indagar las características del conflicto mapuche, dado que no es posible diseñar una matriz que desconozca el centro de la problemática que se pretende estudiar. Teniendo en cuenta que esta etapa del procedimiento analítico de los encuadres es fundamental para el artículo, la retomaremos y profundizaremos en la próxima sección.

Las operaciones de recolección y procesamiento de esos datos resultan novedosas respecto de otros trabajos empíricos que analizan la cobertura mediática de asuntos públicos. La preocupación de estos autores se centra en encontrar un método preciso que reduzca los márgenes de subjetividad del investigador. Para ello, recorren críticamente cinco abordajes metodológicos, que no deben ser entendidos como excluyentes: el hermenéutico, el lingüístico, el holístico-manual, el informatizado y el deductivo. Finalmente, Matthes y Kohring (2008) proponen su propio método de análisis, que consideran superador a la luz de los niveles de confiabilidad y validez que es capaz de conseguir. Si bien aquí consideramos que dicho método es sumamente productivo para el análisis de los encuadres, disentimos con la conceptualización de confiabilidad sugerida por ellos como argumento central para distinguirse de los otros cinco métodos. La subjetividad no es necesariamente un problema para alcanzar la fiabilidad requerida por este procedimiento; por el contrario, para llevar a cabo un análisis de contenido resulta imposible eludir la intervención subjetiva del codificador y/o analista. Más aun, la consideramos constitutiva de este tipo de estudios.

Para la detección de los frames, los autores introducen una técnica de análisis computarizado que permite identificar las variables latentes (Igartua, 2006) en los textos mediante el agrupamiento sistemático de indicadores manifiestos. A diferencia de los abordajes deductivos, que buscan constatar la presencia de encuadres genéricos, este procedimiento permite detectar la emergencia de nuevos frames. La interpretación y la contextualización de tales marcos es llevada a cabo por el analista, que necesita tener una mirada comprensiva del contexto sintáctico y semántico en el que los términos e ideas cobran sentido. 
Dado que el objetivo de este trabajo contempla un planteo teórico-metodológico para la creación y detección de los encuadres del conflicto mapuche en la prensa chilena, en el próximo apartado desarrollamos —ejerciendo una constante vigilancia epistemológica- la desagregación del concepto de encuadre propuesto por Entman (2004) que aplicaremos a nuestro objeto. De dicha operacionalización surgirán cuatro funciones o frame elements: definición del problema, atribución causal, evaluación moral y recomendación de tratamiento.

\subsection{Los encuadres del conflicto mapuche}

En la literatura sobre Framing predominan dos grandes estrategias metodológicas para la detección y análisis de los encuadres mediáticos. Por un lado, aquellas que se enfocan en la aplicación deductiva de encuadres genéricos creados en estudios previos. Por otro, los que crean encuadres específicos a partir del abordaje inductivo de un corpus de noticias. En los primeros, los encuadres son un punto de partida cuya presencia es comprobada en la instancia empírica. En los segundos, los frames resultan del análisis de los datos. Nuestra propuesta se ubica en la intersección de ambas perspectivas. Partiendo de la operacionalización de una definición genérica, las variables y categorías que conforman los elementos de encuadre deben estar en diálogo con las dinámicas del propio caso. En otras palabras, los frame elements son producto de la desagregación del concepto de encuadre a partir de un razonamiento deductivo. En cambio, la identificación de los tópicos, los actores, la dicotomía de universos que definen la evaluación moral del tema mapuche y las acciones propuestas para resolver dichas disputas de sentido quedan plasmadas en variables específicas que surgen de una inducción a los rasgos del caso.

Considerando que la mayoría de las definiciones de encuadre son ciertamente vagas $y$, por lo tanto, no pueden traducirse en indicadores empíricos de manera directa (Matthes y Kohring, 2008), partimos de la definición de Entman, considerada productiva para estructurar las dimensiones de análisis incorporadas a la matriz.

(Encuadrar) implica seleccionar y realzar algunos aspectos de eventos o temas, y hacer conexiones entre ellos para promover una interpretación, evaluación y/o solución. Las palabras e imágenes que componen un encuadre pueden ser distinguidas de las demás noticias por su capacidad para estimular apoyo u oposición a los distintos campos de un conflicto político (Entman, 2003: 417).

De la definición de Entman es posible extraer una serie de ejes que, a su vez, sean operacionalizados en variables directamente observables en las noticias del conflicto mapuche publicadas en periódicos chilenos. En este sentido, la acción de encuadrar consiste en: 1) definir efectos o condiciones como problemáticas; 2) identificar sus causas; 3 ) emitir un juicio moral y; 4) promover un remedio o emitir un pronóstico. 
El primer elemento de encuadre, la definición del problema, apunta a la "definición de la situación" (Goffman, 2006 [1974]: 1) como respuesta a la pregunta ¿qué sucede aquí? Nombrar una situación implica caracterizar una escena, roles a cargo de las actuaciones y actores legitimados para interpretar lo que sucede. Por ejemplo, denominar los incendios que se produjeron en las compañías forestales ubicadas en la Araucanía como "ataques incendiarios" o "actos terroristas" supone una definición de la situación que califica, de modo implícito, a los integrantes de la Coordinadora Arauco Malleco (CAM) como actores responsables de esos acontecimientos. Más aun, esa definición de la situación expresada en las notas periodísticas es coherente con la que sostienen fuentes oficiales como Carabineros y otros funcionarios de los distintos niveles de Gobierno. Ello da cuenta de la capacidad de las voces institucionales de obtener crédito en las coberturas (Koziner, 2017b).

La identificación de causas alrededor de una situación problemática puede relacionarse con la atribución de responsabilidades. Desde la teoría de la atribución, Heider (1964) analizó que los seres humanos no logran entender la complejidad del mundo en que viven y procuran, por lo tanto, inferir sus relaciones causales. En términos individuales, las personas tienden a atribuir la conducta a causas internas o disposicionales. En términos sociales, a causas externas o situacionales.

Tal como mencionamos más arriba, la definición de los delitos contra las compañías forestales como "ataques incendiarios" señala a los miembros de las comunidades Mapuche como responsables aun cuando no se los presente explícitamente como los causantes de la acción relatada. Ello confirma la hipótesis de Entman (2004) según la cual para que un frame tenga resonancia cultural es preciso que resulten visibles, al menos, dos de las cuatro funciones mencionadas: definición del problema y propuesta de tratamiento o pronóstico. En otras palabras, no es necesario explicitar todos los frame elements pues de la definición de la situación se desprenden sus agentes causales y una evaluación moral.

El tercer componente de la definición de encuadre es la evaluación moral. Es decir, la emisión de prescripciones morales que ponderan el problema. Por lo general, estos son medidos en términos de valores compartidos. Un juicio moral es entendido aquí como un dictamen que distingue aquello que, en última instancia, debe ser considerado bueno, así como los deberes que ello implica, de lo que queda asociado a lo malo (Schmitt, 2009), ya sea para un actor individual o para el conjunto de la sociedad.

La forma de operacionalizar esta dimensión consiste en presentar las miradas de los actores en conflicto, estructuradas alrededor de universos dicotómicos en torno a cinco grandes ejes temáticos que hacen a la vida de la sociedad chilena —así como la puja por instalar y cristalizar determinados encuadres para definir esas situaciones-y organizan el debate sobre la cuestión mapuche en la prensa: el eje político, el judicial, el económico, el social y el cultural. 
El eje político se refiere a los derechos políticos que el Estado chileno les reconoce a las comunidades Mapuche. Estos pueden ser expresados de tres maneras distintas. Dos de ellas suponen considerar a estas comunidades en la formulación de políticas públicas a partir de su incorporación en los términos del propio Estado. Una contempla la posibilidad de integrarlos, al reconocerlos como chilenos, y la otra la posibilidad de incluirlos, respetando sus particularidades. La tercera opción, radicalmente distinta a las dos anteriores, sería reconocer su autonomía, es decir, su capacidad de tomar decisiones propias en determinadas materias que exceden la política pública.

El eje judicial alude a los modos en que el Estado chileno gestiona y da solución al conflicto con las comunidades Mapuche. La puja alrededor del reconocimiento o no de sus derechos puede derivar en un diálogo político, que contemple su autonomía y legitime su idiosincrasia; o en una judicialización, que convoque la intervención de otros poderes del Estado para el procesamiento o condena del reclamo de estas comunidades. En el marco de esta dimensión se promueve una interpretación polarizada, que opone la negociación -y salida política- entre los diferentes actores en disputa, a la intervención del Poder Judicial para su resolución:

Tanto la figuración de los personajes principales de la noticia como el modo en que es configurado el acontecimiento delictivo parecen, efectivamente, propiciar la construcción de un universo dicotómico de polaridad del tipo bien/mal o legalidad/ilegalidad. Semejante mirada simplista (...) muchas veces deriva en un sesgo de normalización, según el cual las instituciones —en particular, las estatales- están para reencauzar cualquier tipo de desviación, y en la propuesta de soluciones fundamentalmente punitivistas (Raimondo, Aruguete y Arri, 2019: 35).

El eje económico se refiere a los fundamentos y a la lógica del desarrollo económico de las comunidades Mapuche. En ese terreno, los enfoques utilizados por la prensa oponen la mirada productivista y lucrativa a otra inserta en la producción antimercantil y de supervivencia. En este discurso polarizado discurren la razón y el fin de la actividad productiva y/o de servicios desempeñados por cualquier actor económico, sean estos mapuches, grandes o medianos empresarios, emprendedores u otros. Derivado de ello, el rol desempeñado por el Estado en lo relativo al desarrollo económico de las comunidades oscila entre el de una ayuda inicial como impulso para el crecimiento y el de un apoyo permanente que asegure la subsistencia.

El eje social se refiere a los fundamentos y a las formas que cobran los reclamos de las comunidades Mapuche por el reconocimiento de sus derechos, en el espacio público-privado. La demanda de estas comunidades en torno a la recuperación del territorio ancestral, la lengua, las costumbres y la cultura, puede encuadrarse en términos de exigencia de un derecho no reconocido o, en cambio, como la 
reivindicación de un derecho histórico que les pertenece y les ha sido quitado. Aquí, la dicotomía se expresa en la calificación de dichas manifestaciones, como violentas o pacíficas, en caso de que alguno de los actores que participen del evento recurra o no a la fuerza. Una vez más, esta contraposición es congruente con la forma en que las autoridades del Estado chileno encuadran públicamente los reclamos. Cabe aclarar que ambas categorías versan alrededor de esa mirada dicotómica coconstruida tanto por el gobierno cuanto por los medios, y adquiere "resonancia cultural" (Entman, 2003: 417) en buena parte de la opinión pública chilena, que garantiza la persistencia y consolidación de este frame a lo largo de tiempo (Reese, 2001).

Si bien esta caracterización tiene implicancias políticas, se trata de hechos que se desarrollan en la arena social. Más aun, el relato de los acontecimientos replica los rasgos característicos de las noticias de delito, inseguridad y violencia. En estos casos, se contrapone el Estado chileno, representado por las fuerzas de seguridad que cumplen con su deber de defensores del orden, a las comunidades Mapuche, condensadas en la figura del delincuente. "El enunciador se alza con la autoridad para trazar la línea que separa el bien del mal, lo comprensible de lo irracional" (Fernández y Tobi, 2009: 61), y es en ese marco que califica dicotómicamente a los actores involucrados en los acontecimientos narrados.

El eje cultural, por último, se refiere a los modos de conceptualizar las costumbres culturales de las comunidades Mapuche en el marco de la polarización idiosincrática que habita y absorbe la sociedad chilena alrededor de esta problemática. El enfoque de la cultura de las comunidades en términos ancestrales supone respetar su cosmovisión, su medicina y su lengua. En contraposición, el encuadre folklorizado propone una construcción descontextualizada y deshistorizada de la cultura mapuche, al recrear sus costumbres desde una mirada externa que exalta lo exótico (Soto, 2005).

A continuación, exponemos la figura 1, donde consta la totalidad de dimensiones, variables y categorías que integran los frames elements de la matriz diseñada para el análisis de la cobertura del conflicto entre las comunidades Mapuche y el Estado chileno. 
Fuente: elaboración propia.

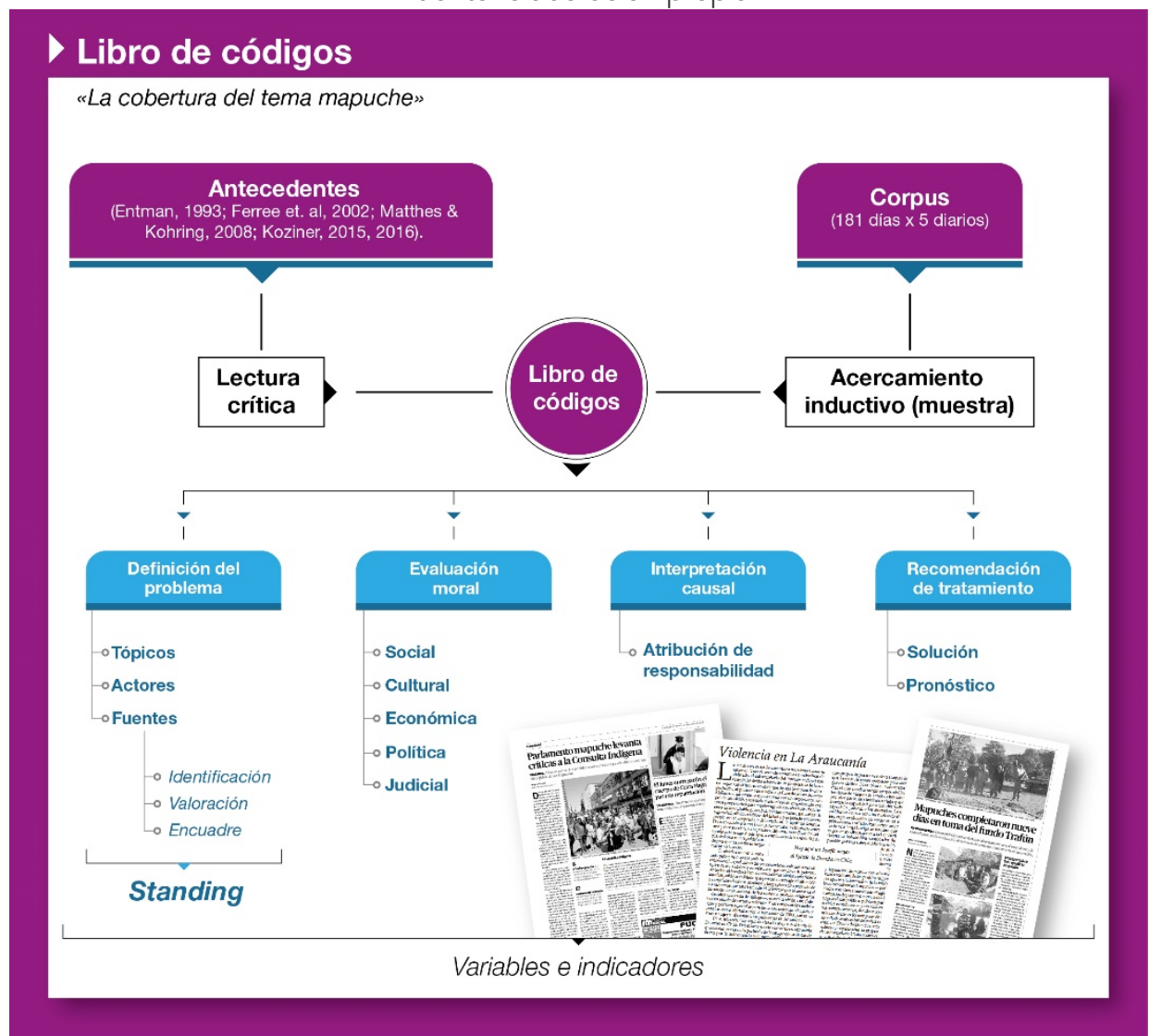

Figura 1. Elementos de encuadre, variables y categorías que componen la matriz de análisis del conflicto mapuche en la prensa chilena

\section{A modo de cierre}

El objetivo de este trabajo fue estructurar un procedimiento analítico para la detección de los encuadres del conflicto mapuche que permita reflexionar acerca del tratamiento que la prensa chilena hizo de este asunto en el período 2010-2012 y 2014-2016. En este marco, nos enfocamos en las bases conceptuales de la matriz, cuyo propósito es analizar la relevancia de este fenómeno en la prensa chilena nacional y regional.

Consideramos al Framing como una aproximación productiva para este propósito, por cuanto se trata de un modelo puente que "une las partes del campo que deben estar en contacto entre sí: es cuantitativo y cualitativo, empírico e interpretativo, psicológico y sociológico, académico y pro-profesional" (Reese, 2007: 148).

La creación de las variables descriptas y los valores o categorías que cada una puede asumir, involucró razonamientos de tipo inductivo y deductivo. La lectura 
crítica de bibliografía se combinó con aquellos datos que surgieron de trabajos previos y con los que se obtuvieron de una aproximación inductiva a una muestra del corpus (Koziner, 2017a). Luego, mediante un ejercicio de elaboración teórica, se procuró incorporar los distintos tipos de datos a la matriz conformada para el análisis de las notas, manteniendo una constante vigilancia epistemológica. Esta última instancia involucró un razonamiento deductivo, pues la matriz será aplicada a la recolección sistemática de los datos de las notas — proceso de codificación, en el marco del análisis de contenido- y al análisis estadístico de los mismos (Sautu et al., 2005).

El procesamiento de los datos recogidos como producto de la codificación permitirá determinar el modo en el que los distintos elementos aparecen agrupados en los textos, dando lugar a los frames. Ello posibilitará identificar, además, la relevancia que el caso cobró en la prensa gráfica durante el periodo analizado y los niveles de acreditación y legitimación de los actores involucrados en los hechos relatados.

En sintonía con las definiciones presentadas más arriba, los frames que emergerán del análisis de los datos pueden caracterizarse como "patrones persistentes de cognición, interpretación y presentación, de selección, énfasis y exclusión, a través de los cuales quienes manejan los símbolos organizan de forma rutinaria el discurso, ya sea verbal o visual" (Gitlin, 2003: 7). Los frames de las voces oficiales y de los actores económica y culturalmente poderosos suelen tener mayor presencia en los contenidos informativos (Gans, 2004), asunto que se corrobora en el análisis de los datos, cuyo objetivo último es conocer el grado de pluralismo de la cobertura de la cuestión mapuche.

Se espera que la elaboración de una matriz de análisis para el estudio de noticias sobre el conflicto mapuche en Chile constituya una herramienta que pueda ser utilizada para el abordaje de los contenidos mediáticos. Su fortaleza radica en su capacidad de ser aplicada sistemáticamente a la totalidad de un corpus susceptible de ser ampliado. La profusa base de datos cuantitativos y cualitativos que surjan de ese proceso colaborará con la descripción, el análisis y la discusión científica en relación con una cuestión de suma relevancia y actualidad para las agendas política, mediática y pública chilenas, pero también para las de carácter regional.

\section{Financiación}

El estudio fue realizado en el marco del proyecto PLU 170002 del Fondo de estudios sobre el pluralismo en el sistema informativo nacional, financiado por la Comisión Nacional de Investigación Científica y Tecnológica (CONICYT) de la República de Chile. 


\section{Referencias bibliográficas}

ARUGUETE, N. y AMADEO, B. (2012): Encuadrando el delito: pánico moral en los periódicos argentinos. América Latina Hoy, no. 62, pp. 177-196. Disponible en: http://www.redalyc.org/resumen.oa?id=30825868008

ARUGUETE, N. y KOZINER, N. (2014): La cobertura mediática del '7D' en la prensa argentina. Aplicación de encuadres noticiosos genéricos a los principales diarios nacionales. Disertaciones. Anuario electrónico de estudios en Comunicación Social, 7 (1), pp. 129-165. Disponible en:

http://150.185.138.216/index.php/Disertaciones/article/view/4536

ENTMAN, R. M. (1993): Framing: Toward Clarification of a Fractured Paradigm. Journal of Communication, 43 (4), pp. 51-58. Doi: http://doi.org/10.1111/j.14602466.1993.tb01304.x

(2003): Cascading Activation: Contesting the White House's Frame After 9/11. Political Communication, 20 (4), pp. 415-432. Doi: http://doi.org/10.1080/10584600390244176.

(2004): Projections of power: Framing news, public opinion, and US foreign policy. Chicago: University of Chicago Press.

FERNÁNDEZ, J. L. y TOBI, X. (2009): Criminal y contexto: estrategias para su figuración. Revista LIS (Letra, Imagen, Sonido) Ciudad Mediatizada, no. 4.

GANS, H. J. (2004): Deciding What's News: A Study of CBS Evening News, NBC Nightly News, Newsweek, and Time (2ed.). Evanston, Illinois: Northwestern University Press.

GITLIN, T. (2003): The Whole World Is Watching Mass Media in the Making and Unmaking of the New Left (2ed.). California: University of California Press.

GOFFMAN, E. (2006): Frame analysis: los marcos de la experiencia. Madrid: Centro de Investigaciones Sociológicas.

HEIDER, F. (1964): The Psychology of Interpersonal Relations (3ed). New York: John Wiley \& Sons, Inc. Doi: http://doi.org/10.1037/10628-000

HOLSTI, O. R. (1969). Content analysis for the social sciences and humanities. Reading, MA: Addison-Wesley.

IGARTUA, J. J. (2006): El análisis de contenido. En IGARTUA, J. J., Métodos cuantitativos de investigación en comunicación (1ed.). Barcelona: Bosch, pp. 175229. 
KOZINER, N. (2017a): La prensa económica y el debate sobre los medios. Análisis de la cobertura informativa del proceso de discusión de la Ley de Servicios de Comunicación Audiovisual en Ámbito Financiero y El Cronista Comercial (marzooctubre de 2009). Tesis de Doctorado no publicada, Universidad Nacional de Quilmes.

(2017b): Periodistas y fuentes en la prensa argentina. Revisión teórica a partir de un caso empírico. Revista Mexicana de Opinión Pública, no. 24, pp. 147-167. Doi: http://doi.org/10.22201/fcpys.24484911e.2018.24.61707

KOZINER, N. y ARUGUETE, N. (2016): El '7D' en los diarios económicos argentinos. Aplicación de encuadres noticiosos genéricos a la cobertura de la controversia judicial entre el Gobierno y el Grupo Clarín. Comunicación y Medios, no. 33, pp. 734. Doi: https://doi.org/10.5354/0719-1529.2016.38389

(2017): Políticas de comunicación en la prensa gráfica argentina. Intersecciones en Comunicación, no. 10, pp. 31-49.

(2020). Pluralismo en la prensa chilena. Propuesta metodológica para el estudio de la cobertura del tema mapuche. En VALLE, C. y VALDIVIA, P. (Eds.): Leyendo el tejido social. Análisis discursivo y retórica cultural en el sur global (1ed.). Temuco: Editorial UFRO, pp. 329-353.

KRIPPENDORFF, K. (1990): Metodología de análisis de contenido. Teoría y práctica. Barcelona: Paidós.

MATTHES, J. y KOHRING, M. (2008): The Content Analysis of Media Frames: Toward Improving Reliability and Validity. Journal of Communication, 58 (2), pp. 258-279.

PIOVANI, J. I. (2018): El diseño de la investigación. En MARRADI, A., ARCHENTI, N. y PIOVANI, J.I.: Manual de Metodología de las Ciencias Sociales. Buenos Aires: Siglo XXI Editores, pp. 81-100.

RAIMONDO, N. R., ARUGUETE, N. y ARRI, F. H. (2019): Apostillas en torno al discurso periodístico sobre delito e inseguridad: figuraciones de las víctimas y los victimarios en noticieros argentinos de televisión abierta. Galáxia (São Paulo), no. 42, pp. 23-39.

REESE, S. D. (2001). Prologue. A bridging model for media research. En S. REESE, O. GANDY, y A. GRANT (Eds.), Framing Public Life: Perspectives on Media and our Understanding of the Social World. Mahwah: Lawrence Erlbaum, pp. 7-31. 
(2007): The framing project: A bridging model for media research revisited. Journal of Communication, 57 (1), pp. 148-154.

SAUTU, R., BONIOLO, P., DALLE, P. y ELBERT, R. (2005): Manual de metodología: construcción del marco teórico, formulación de los objetivos y elección de la metodología. Buenos Aires: CLACSO Campus Virtual.

SCHMITT, C. (2009): El concepto de lo político. Madrid: Alianza Editorial. Doi: https://doi.org/10.1007/s13398-014-0173-7.2

SOTO, S. (2005): La delincuencia en la agenda mediática. Reis, 112 (5), pp. 75-130.

VALLE, C. (2005): Interculturalidad e intraculturalidad en el discurso de la prensa: Cobertura y tratamiento del discurso de las fuentes en el 'conflicto indígena mapuche', desde el discurso político. Redes, no. 2, pp. 83-111. 